\section{Displasia arritmogénica del ventrículo derecho con compromiso de ventrículo izquierdo: valor diagnóstico de la resonancia cardiaca}

\author{
RIENZI DÍAZ ${ }^{1,2}$, DANILO SILVA ${ }^{3}$
}

Cardiac magnetic resonance in arrhythmogenic right ventricular dysplasia. Report of two cases

Arrhythmogenic right ventricular dysplasia is an inherited condition characterized by replacement of normal myocardium by fatty or fibro-fatty tissue, which mainly affects the right ventricle. The most frequent form of presentation is ventricular tachycardia or sudden death, whose origin is considered to be a product of fibrous or fatty infiltration of the myocardium. This structural damage can be detected by cardiac magnetic resonance imaging (MR). We report two patients with ventricular tachycardia due to arrhythmogenic right ventricular dysplasia. A 49 year-old female with a history of ventricular tachycardia. EKG showed epsilon waves and a prolonged QTc. Echocardiogram showed right ventricular dilatation and dysfunction. MR showed right ventricular fatty infiltration. An implantable cardioverter-defibrillator was installed to the patient. A 37 year-old male was admitted for recurrent syncope. On admission a ventricular tachycardia was detected. An echocardiogram showed right ventricular dilatation and dysfunction. MR showed a large zone of fibrosis in the right ventricle. An implantable cardioverter-defibrillator was also installed.

(Rev Med Chile 2014; 142: 1467-1472)

Key words: Arrythmogenic right ventricular dysplasia; Magnetic resonance imaging; Cardiomyopathies; Tachycardia, ventricular.
${ }^{1}$ Departamento de Enfermedades

Cardiovasculares, Escuela de Medicina, Universidad de

Valparaíso.

${ }^{2}$ Departamento de Cardiología

y Cirugía Cardiovascular, Clínica Reñaca, Chile.

${ }^{3}$ Unidad de Cuidados Intensivos

Adultos, Clínica Reñaca, Chile.

Recibido el 8 de diciembre de 2013, aceptado el $1^{\circ}$ de octubre de 2014.

Correspondencia a:

Dr. Rienzi Díaz Navarro Profesor Adjunto de Cardiología, Universidad de Valparaíso. 4 Poniente 332, Of. 305, Viña del Mar, Chile. Teléfono: 56-32-2658525

Fax: 56-32-2658527 diaz.rienzi@gmail.com
L a displasia arritmogénica del ventrículo derecho (DAVD) es una miocardiopatía hereditaria y progresiva que compromete principalmente el ventrículo derecho $(\mathrm{VD})^{1-3}$, pero también puede afectar el ventrículo izquierdo (VI) aumentando su severidad ${ }^{1,2,4,5}$. Histopatológicamente se caracteriza por reemplazo fibrograso del tejido miocardio que produce un daño estructural progresivo $^{4,6}$, manifestado comúnmente por taquicardia ventricular (TV) y muerte súbita ${ }^{4,7}$.

Producto de la complejidad de esta enfermedad, el Grupo de Trabajo para DAVD de la Sociedad Europea de Cardiología propuso criterios diagnósticos electrocardiográficos, arrítmicos, histopatológicos, genéticos y morfo funcionales, internacionalmente aceptados ${ }^{8}$. Para aumentar su sensibilidad diagnóstica, estos criterios fueron recientemente modificados, incluyéndose hallazgos obtenidos en resonancia cardiaca $(\mathrm{RC})^{9,10}$. Este método de diagnóstico no-invasivo permite evaluar con gran precisión la anatomía, función y características del tejido miocárdico de ambos ventrículos ${ }^{11,12}$.

Comunicamos dos casos en los que la RC juega un papel clave para diagnóstico de DAVD, identificando de manera no-invasiva la infiltración 
fibrograsa característica de esta enfermedad y el compromiso del VI.

\section{Casos clínicos}

\section{Caso 1}

Mujer de 49 años con historia de TV recurrente autolimitada referida para RC por sospecha de DAVD. Sin historia familiar de cardiopatía y exámenes de laboratorio normales. El electrocardiograma de reposo (ECG) mostró ondas épsilon (flechas negras), ondas T (-) de V1 a V4, y QT prolongado (QTc $=510 \mathrm{~ms}$ ) (Figura 1A). El ecocardiograma mostró un VD con diámetro diastólico $30 \mathrm{~mm}$, disfunción ventricular moderada sin alteración segmentaria, ni formaciones aneurismáticas.

La RC con secuencias de eco-gradiente en 4-cavidades (Figuras 1B y 1C) y eje corto (Figuras $1 \mathrm{E}$ y $1 \mathrm{~F}$ ), mostró dilatación del ventrículo derecho [VDVD/ASC $115 / \mathrm{m}^{2}$ ] con disfunción ventricular severa (FE 14\%), microaneurismas en la pared lateral del VD (flechas amarillas) y zonas disquinéticas (flechas azules) en pared lateral (Figura 1C), pared anterior e inferior (Figura $1 F)$. Además, infiltración grasa en la región latero apical del VI (flecha verde). El VI tenía volúmenes internos normales y zona aquinética posterolateral con miocardio adelgazado (flechas rojas). En la imagen con secuencia potenciada en T1 (Figura 1D), se observó infiltración grasa en la región subepicárdica y miocardio de la pared lateral del VD y ápex, así como en la región lateroapical del VI (flechas verdes).

Los hallazgos descritos confirman el diagnóstico de DAVD con compromiso de VI en presencia de dos criterios diagnósticos mayores: onda épsilon en derivaciones precordiales V1 a V4; disquinesia regionales del VD más cociente de volumen telediastólico del $\mathrm{VD}>100 \mathrm{ml} / \mathrm{m}^{2}$ (mujeres) y fracción de eyección (FE) del $\mathrm{VD}<40 \%$. El tratamiento dela paciente incluyó la implantación de un cardiodesfibrilador (CDI).

\section{Caso 2}

Hombre de 37 años, fumador de 60 cigarrillos/día desde su adolescencia, hospitalizado por síncope recurrente. Al ingreso, se registró TV monoforma con frecuencia de $233 \mathrm{lpm}$ en trazado de monitor (Figura 2A), que convirtió espontá- neamente a ritmo sinusal (Figura 2B). El paciente fue consumidor ocasional de cocaína hasta 3 años antes del ingreso. Negó tener angina o disnea. En el ECG post-conversión a ritmo sinusal se observó ondas $\mathrm{T}(-)$ en las derivaciones precordiales y de pared inferior (Figura 2B). La troponina I alcanzó un valor máximo de $0,8 \mathrm{ng} / \mathrm{ml}[\mathrm{VN}<0,4 \mathrm{ng} / \mathrm{ml}]$. Un ecocardiograma reveló leve aumento de los diámetros internos del VI, disquinesia apical y lateroapical con función sistólica global levemente disminuida [FE 45\%, Simpson]. En la evaluación del VD no se encontró anormalidades. Con el diagnóstico presuntivo de enfermedad coronaria, se realizó estudio angiográfico que mostró arterias coronarias normales. Para avanzar en el estudio diagnóstico, se solicitó RC. Las imágenes con secuencias de eco-gradiente en 4-cavidades (Figura 2C) y eje corto (Figura 2E), mostraron dilatación del VD [VDVD/ASC $122 / \mathrm{m}^{2}$ ] con acentuada disminución del espesor parietal especialmente en pared lateral, microaneurismas (flechas amarillas), disquinesia de pared lateral basal e inferior (flechas azules) y función global severamente disminuida (FE 19\%). Al mismo tiempo, se observó leve aumento de los diámetros internos del VI con moderada diminución de su función sistólica global (FE 38\%), dos zonas con disquinesia en la región apical con adelgazamiento de la pared miocárdica (flechas azules) y una zona de aquinesia con miocardio adelgazado en pared lateral (flecha roja) e infiltración grasa adyacente (flecha verde). En la imagen axial potenciada en T1 (Figura 2F), se observa infiltración grasa subepicárdica en pared lateral del VD, y la pared lateral y ápex del VI (flechas verdes). A su vez, la imagen de 4-cavidades con secuencias de inversiónrecuperación para evaluar viabilidad, luego de la administración de gadolinio endovenoso (Figura 2D), mostró extensa área de fibrosis (realce tardío) en la pared lateral del VD y región subtricuspídea (flechas naranjas).

Los hallazgos descritos son diagnósticos para DAVD con compromiso de VI, en presencia de tres criterios diagnósticos mayores: ondas $\mathrm{T}$ invertidas en derivaciones precordiales derechas (V1 a V6) en individuo > 14 años sin bloqueo de rama derecho, disquinesia regionales del VD más cociente de volumen telediastólico del VD $>110 \mathrm{ml} / \mathrm{m}^{2}$ (varones); TV documentada. El tratamiento de la paciente incluyó la implantación de un CDI. 
Displasia arritmogénica del ventrículo derecho - R. Díaz et al
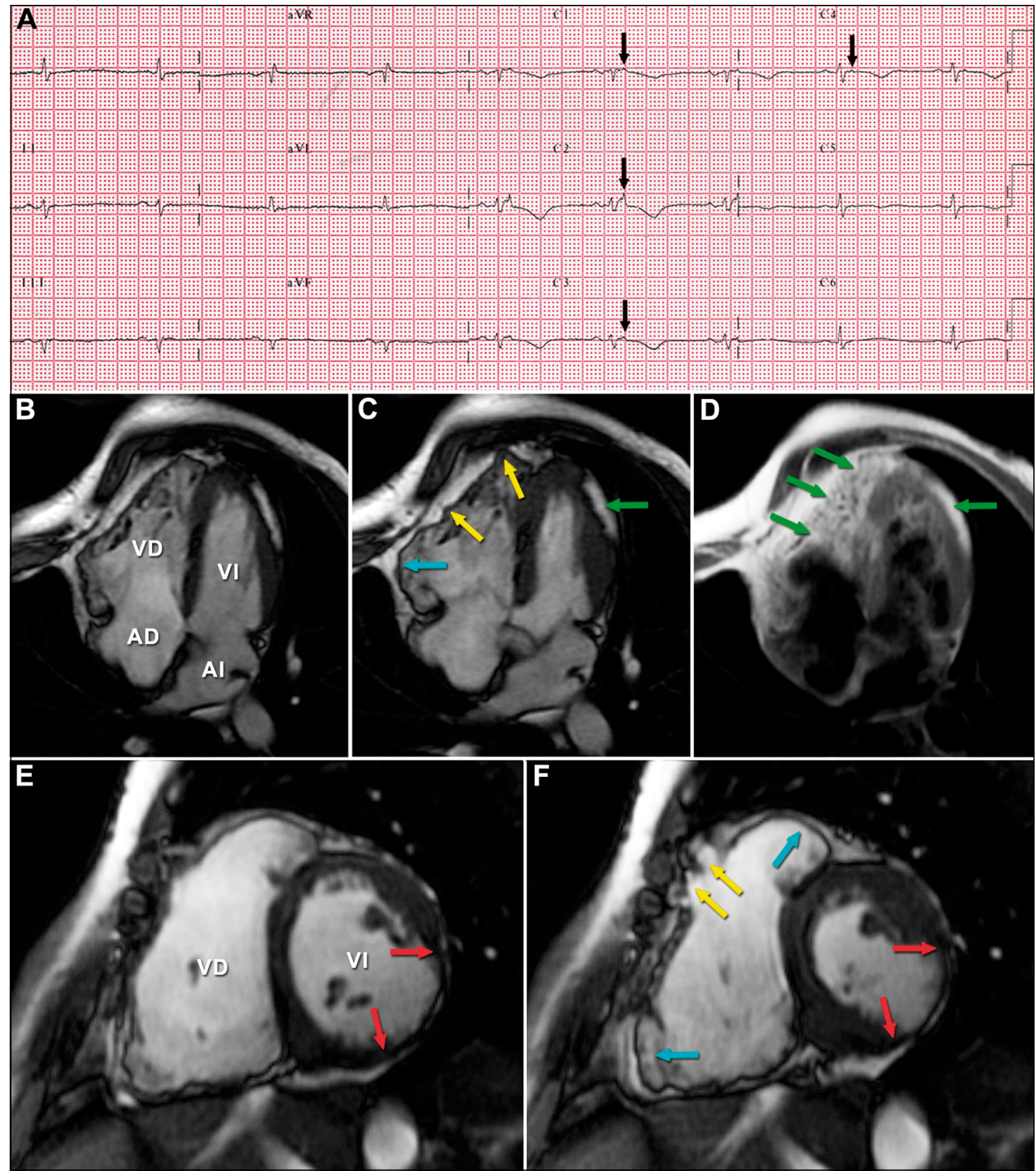

Figura 1. (A) Electrocardiograma de 12 derivaciones muestra inversión de onda T desde $\mathrm{V} 1$ a $\mathrm{V} 5$, ondas épsilon de $\mathrm{V} 1$ a V4 (flechas negras) e intervalo QT prolongado (QTC = 510 ms). Imágenes de RC con secuencia de eco-gradiente en visión de 4-cavidades (B, diástole; $\mathbf{C}$, sístole) y eje corto (E, diástole; $\mathbf{F}$, sístole), muestran marcada dilatación del VD y presencia de microaneurismas (flechas amarillas) y zonas disquinéticas (flechas azules) localizadas en pared lateral, anterior e inferior. Además, infiltración grasa en la región lateroapical del VI (flecha verde). EI VI tiene volúmenes internos en rango normal con un área de aquinesia posterolateral acompañada de miocardio adelgazado (flechas rojas). (D) Imagen de RC con secuencias potenciadas en T1, revela la existencia de infiltración grasa que compromete la región subepicárdica y miocardio de la pared libre del VD y ápex, así como la región lateroapical del VI (flechas verdes). AD, aurícula derecha; $\mathrm{Al}$, aurícula izquierda; $\mathrm{RC}$, resonancia cardiaca; VD, ventrículo derecho; VI, ventrículo izquierdo. 

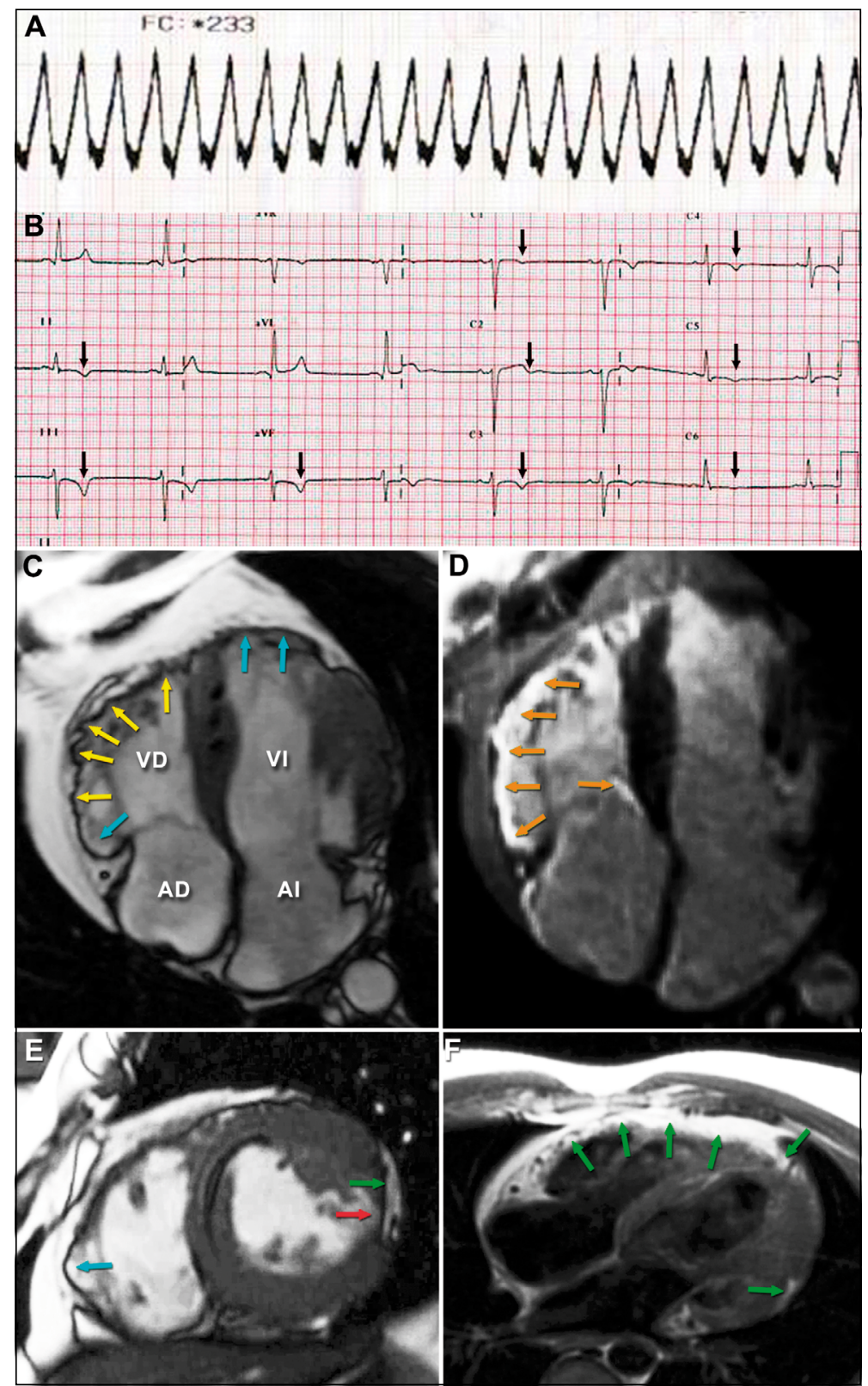

Figura 2. (A) Trazado electrocardiográfico de monitor de 1 derivación, muestra taquicardia ventricular monomorfa de 233 Ipm. (B) electrocardiograma de 12 derivaciones post-cardioversión espontánea a ritmo sinusal, muestra ondas T (-) en derivaciones precordiales y de pared inferior (flechas negras). Imágenes de RC con secuencia de eco-gradiente, visión de 4-cavidades (C) y eje corto (E), en sístole muestran marcada dilatación del VD con numerosos microaneurismas en pared lateral y anterior (flechas amarillas) y disquinesia de pared lateral basal e inferior (flechas azules). El VI tiene leve aumento de sus volúmenes internos, dos zonas de disquinesia en la región apical (flechas azules), un área de aquinesia en la pared lateral con adelgazamiento del miocardio (flecha roja) e infiltración grasa adyacente (flecha verde). (D) Imagen de RC, visión de 4-cavidades, con secuencias de inversión-recuperación para evaluar viabilidad luego de la administración de gadolinio endovenoso, revela extensa área de fibrosis (realce tardío) en la pared lateral del VD y región subtricuspídea (flechas naranjas). (F) Imagen axial de RC con secuencia potenciada en T1, muestra la existencia de infiltración grasa sub-epicárdica en la pared lateral del VD, ápex y pared lateral del VI (flechas verdes). AD, aurícula derecha; Al, aurícula izquierda; RC, resonancia cardiaca; VD, ventrículo derecho; VI, ventrículo izquierdo. 


\section{Discusión}

La DAVD se presenta comúnmente por palpitaciones, fatiga y síncope. En algunos casos, la muerte súbita post esfuerzo puede ser la primera manifestación ${ }^{4,7}$ y muy ocasionalmente debuta como un síndrome coronario agudo ${ }^{13}$.

La elevada mortalidad inherente a la DAVD amerita que el diagnóstico sea establecido lo más precozmente posible, permitiendo optimizar su manejo con el objeto de prevenirla muerte súbita.

Una de las características electrocardiográficas típicas de la DAVD es la presencia de ondas épsilon, que representan potenciales eléctricos post-excitación de baja amplitud, registrados al final del complejo QRS (Figura 2D) y constituyen un criterio diagnóstico electrocardiográfico mayor para DAVD ${ }^{4,7,14,15}$, hallazgo presente en nuestra primera paciente (Figura 1A). Estas ondas se originan en zonas de tejido sano rodeadas por tejido fibrograso y exteriorizan áreas de conducción lenta que gatillan la generación de arritmias ventriculares por re-entrada.

Por otra parte, el ecocardiograma es una herramienta de gran utilidad diagnóstica, que eventualmente permite objetivar la existencia de alteraciones segmentarias de la contracción (ASC) del VD, las que asociadas a dilatación ventricular derecha o cambios del área fraccional del VD $\leq 33 \%$, constituyen criterios ecocardiográficos mayores para el diagnóstico de esta afección ${ }^{9,10}$. Sin embargo, siendo la ecocardiografía una técnica útil, tiene limitaciones para la valoración de las ASC del VD, incluso en manos experimentadas y más allá de las relacionadas con ventanas ecocardiográficas sub-óptimas ${ }^{17}$. De hecho, la ecocardiografía bidimensional en nuestros pacientes sólo mostró leve dilatación del VD en el primer caso y un VI levemente dilatado con aquinesia apical en el segundo, ninguno de los cuales constituyen criterios diagnósticos mayores para DAVD ${ }^{9,10}$.

Durante mucho tiempo, la ventriculografía ha sido considerada el patrón diagnóstico de oro para la DAVD, porque puede complementarse con la toma de biopsias para localizar la infiltración fibrograsa, pero es una técnica invasiva no exenta de complicaciones. En la actualidad, no parece necesaria su realización rutinaria al existir la RC que permite identificar la infiltración fibrograsa intramiocárdica ${ }^{11,12}$, como ocurrió en nuestros pacientes (Figura 1D y Figura 2F). A su vez, la RC con contraste-luego de la administración de gadolinio endovenoso-permite descubrir la presencia de fibrosis que se manifiesta como híper-realce en imágenes tardías post-gadolinio, propiedad observada en el segundo caso (Figura 2D). Por lo tanto, la RC debe ser ahora considerada la técnica imagenológica de elección para la valoración diagnóstica de pacientes con sospecha de DAVD ${ }^{4,11}$. En la modificación de los criterios diagnósticos para $\mathrm{DAVD}^{9,10}$, los siguientes hallazgos en RC constituyen criterios diagnósticos mayores: áreas de aquinesia, disquinesia o contracción disincrónica de VD, asociadas a dilatación ventricular con volumen final de diástole indexado $\geq 110 \mathrm{ml} / \mathrm{m}^{2}$ en varones $\mathrm{y} \geq 100 \mathrm{ml} / \mathrm{m}^{2}$ en mujeres, $\mathrm{y} / \mathrm{o}$ disfunción de $\mathrm{VD}[\mathrm{FE} \leq 40 \%]$, características presentes en ambos casos.

Además de los criterios diagnósticos por $\mathrm{RC}$, nuestros pacientes tenían también criterios electrocardiográficos mayores para DAVD ${ }^{9,10}$. El ECG en la primera paciente registró ondas épsilon (Figura 1A) y en el segundo ondas $\mathrm{T}$ invertidas en todas las derivaciones precordiales y de pared inferior (Figura 2B), que en ausencia de bloqueo completo de rama derecha en un paciente $>14$ años, también es criterio diagnóstico mayor para DAVD.

De acuerdo a los nuevos criterios diagnósticos para la DAVD, el diagnóstico definitivo requiere presencia de dos criterios mayores o un criterio mayor y dos menores, o cuatro criterios menores de categorías diagnósticas diferentes ${ }^{18}$.

El objetivo del tratamiento de la DAVD es el manejo adecuado de la TV y la prevención de la muerte súbita. Los beta-bloqueadores y la amiodarona siguen siendo el tratamiento de base, sin embargo, para la prevención de la muerte súbita la implantación de un CD es mandatoria ${ }^{19}$.

Por otra parte, la identificación de fibrosis por $\mathrm{RC}$ es un predictor de inducción de TV en estudios electrofisiológicos al tener propiedades arritmogénicas ${ }^{11}$. A su vez, la ausencia de realce tardío en algunos casos, puede explicarse por la presencia exclusiva de infiltración grasa o con escasa fibrosis que impide su pesquisa en la RC. En consecuencia, la posibilidad de objetivar fibrosis en los estadios tempranos de la enfermedad es relevante, porque permite el manejo oportuno y por ende prevenir la muerte súbita.

Por último, es indispensable mencionar que en el estudio de pacientes con TV de VD, en los cuales 
la RC no es concluyente para DAVD, los estudios genéticos pueden ser la herramienta diagnóstica al identificar una mutación patógena asociada, o probablemente asociada a DAVD ${ }^{20,21}$.

Los casos clínicos comunicados ilustran el rol clave que tiene la RC para diagnóstico definitivo de DAVD, identificando de manera no invasiva características estructurales y morfofuncionales de esta grave enfermedad cardiaca.

\section{Referencias}

1. Hulot JS, Jouven X, Empana JP, Frank R, Fontaine G. Natural History and Risk Stratification of Arrhythmogenic Right Ventricular Dysplasia/Cardiomyopathy. Circulation 2004; 110: 1879-188.

2. Dalal D, Nasir K, Bomma C, Prakasa K, Tandri H, Piccini J, et al. Arrhythmogenic Right Ventricular Dysplasia: a United States Experience. Circulation 2005; 112: 382332.

3. Basso C, Corrado D, Marcus F, Nava A, Thiene G. Arrhythmogenic right ventricular dysplasia. Lancet 2009; 373: 1289-300.

4. Corrado D, Basso C, Thiene G, McKenna WJ, Davies MJ, Fontaliran F, et al. Spectrum of Clinicopathological Manifestations of Arrhythmogenic Right Ventricular Cardiomyopathy/Dysplasia: A Multicenter Study, J Am Coll Cardiol 1997; 30: 1512-20.

5. Pinamonti B, Sinagra G, Salvi A, Di Lenarda A, Morgera $\mathrm{T}$, Silvestri $\mathrm{F}$, et al. Left ventricular involvement in right ventricular dysplasia. Am Heart J 1992; 123: 711-24.

6. Thiene G, Nava A, Corrado D, Rossi L, Pennelli N. Right Ventricular cardiomyopathy Sudden Death in Young People. N Eng J Med 1988; 318: 129-33.

7. Basso C, Corrado D, Thiene G. Arrhythmogenic Right Ventricular Cardiomyopathy in athletes: Diagnosis, Management and Recommendations for Sport Activity. Cardiol Clin 2007; 25: 415-42.

8. McKenna WJ, Thiene G, Nava A, Fontaliran F, Blomstrom-Lundqvist C. Diagnosis of arrhythmogenic right ventricular dysplasia/cardiomyopathy. Br Heart J 1994; 71:215-8.

9. Marcus FI, McKenna MJ, Sherrill D, Basso C, Bauce B, Bluemke DA, et al. Diagnosis of Arrhythmogenic Right Ventricular Cardiomyopathy/Dysplasia: Proposed Modification of the Task Force Criteria. Eur Heart J 2010; 31: 806-14.

10. Marcus FI, McKenna MJ, Sherrill D, Basso C, Bauce B, Bluemke DA, et al. Diagnosis of Arrhythmogenic Right
Ventricular Cardiomyopathy/Dysplasia: Proposed Modification of the Task Force Criteria. Circulation 2010; 121: 1533-41.

11. Tandri H, Calkins H, Nasir K, Bomma C, Castillo E, Rutberg J, et al. Magnetic Resonance Imaging Findings in Patients Meeting Task Force Criteria for Arrhythmogenic Right Ventricular Dysplasia. J Cardiovasc Electrophysiol 2003; 14: 476-82.

12. Tandri H, Saranathan M, Rodríguez ER, Martínez C, Bomma C, et al. Noninvasive Detection of Myocardial Fibrosis in Arrhythmogenic Right Ventricular Cardiomyopathy Using Delayed-Enhancement Magnetic Resonance Imaging. J Am Coll Cardiol 2005; 45: 98-103.

13. Khalil SI, Kamal A, Ahmad S. Arrhythmogenic right ventricular dysplasia presenting as acute coronary syndrome: a case report. Eur J Echocardiography 2004; 5: 394-8.

14. Marcus FI, Guiraudon G, Frank R, Laurenceau JL, Malergue C, Grosgogeat Y. Right ventricular dysplasia: a report of 24 adult cases. Circulation 1982; 65: 384-98.

15. Fisher NG, Gilbert TJ. Arrhytmogenic right ventricular dysplasia. An illustrated review highlighting developments in the diagnosis and management of this potentially fatal condition. Postgrad Med J 2000; 76: 395-8.

16. Blomström-Lundqvist $C$, Beckman-Suurküla $M$, Wallentin I, Jonsson R, Olsson SB. Ventricular dimensions and wall motion assessed by echocardiography in patients with arrhythmogenic right ventricular dysplasia. Eur Heart J 1988; 9: 1291-302.

17. Yoerger DM, Marcus F, Sherrill D, Calkins H, Towbin JA. Echocardiographic Findings in Patients Meeting Task Force Criteria for Arrhythmogenic Right Ventricular Dysplasia. J Am Coll Cardiol 2005; 45: 860-5.

18. Quarta G, Elliott PM. Criterios diagnósticos para la miocardiopatía arritmogénica del ventrículo derecho Rev Esp Cardiol 2012; 65: 599-605.

19. Corrado D, Leoni L, Link MS, Della Bella P, Gaita F, Curnis A, et al. Implantable Cardioverter-Defibrillator Therapy for Prevention of Sudden Death in Patients with Arrhythmogenic Right Ventricular Cardiomyopathy/Dysplasia. Circulation 2003; 108: 3084-91.

20. Campuzano O, Alcalde M, Allegue C, Iglesias A, GarcíaPavía P, Partemi S, et al. Genetics of arrhythmogenic right ventricular cardiomyopathy. J Med Genet 2013; 50: 280-9.

21. Marcus FI, Edson S, Towbin JA. Genetics of Arrhythmogenic Right Ventricular Cardiomyopathy. A Practical Guide for Physicians. J Am Coll Cardiol 2013; 61: 19458 . 